$10-1-2013$

\title{
Cardiac risk factors and risk scores vs cardiac computed tomography angiography: a prospective cohort study for triage of ED patients with acute chest pain.
}

\author{
Ethan J Halpern \\ Thomas Jefferson University \\ Jacob P Deutsch \\ Thomas Jefferson University \\ Maria M Hannaway \\ Thomas Jefferson University \\ Adrian T Estepa

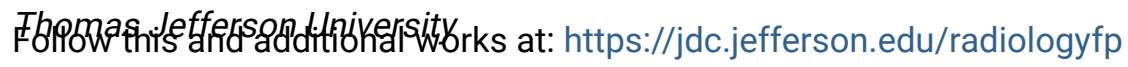 \\ aradtSoKtemiaradiology Commons \\ Theras is fefferso Wwiversitw access to this document benefits you
}

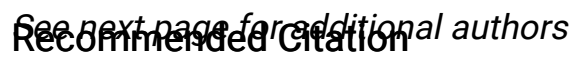

Halpern, Ethan J; Deutsch, Jacob P; Hannaway, Maria M; Estepa, Adrian T; Kenia, Anand S; Neuburger, Kenneth J; and Levin, David C, "Cardiac risk factors and risk scores vs cardiac computed tomography angiography: a prospective cohort study for triage of ED patients with acute chest pain." (2013). Department of Radiology Faculty Papers. Paper 30.

https://jdc.jefferson.edu/radiologyfp/30

This Article is brought to you for free and open access by the Jefferson Digital Commons. The Jefferson Digital Commons is a service of Thomas Jefferson University's Center for Teaching and Learning (CTL). The Commons is a showcase for Jefferson books and journals, peer-reviewed scholarly publications, unique historical collections from the University archives, and teaching tools. The Jefferson Digital Commons allows researchers and interested readers anywhere in the world to learn about and keep up to date with Jefferson scholarship. This article has been accepted for inclusion in Department of Radiology Faculty Papers by an authorized administrator of the Jefferson Digital Commons. For more information, please contact: JeffersonDigitalCommons@jefferson.edu. 


\section{Authors}

Ethan J Halpern, Jacob P Deutsch, Maria M Hannaway, Adrian T Estepa, Anand S Kenia, Kenneth J Neuburger, and David C Levin 


\title{
As submitted to:
}

\author{
American Journal of Emergency Medicine
}

And later published as:

\section{Cardiac Risk Factors and Risk Scores versus Cardiac CTA for Triage of Low and Intermediate Risk Patients with Acute Chest Pain}

\section{October 2013, Volume 31, Issue 10, pp. 1479-85 \\ DOI: 10.1016/j.ajem.2013.08.001}

\begin{abstract}
Objective: To evaluate cardiac risk factors and risk scores for prediction of coronary artery disease $(\mathrm{CAD})$ and adverse outcomes in an emergency department (ED) population judged to be at low to intermediate risk for acute coronary syndrome (ACS).

Methods: This prospective study was approved by the institutional review board. Written informed consent was obtained from consecutive ED patients who presented with chest pain and were evaluated with coronary CTA (cCTA). Cardiac risk factors, clinical presentation, ECG and laboratory studies were recorded; TIMI and GRACE scores were tabulated. cCTA findings were rated on a 6 level plaque burden scale and classified for significant CAD (stenosis $\geq 50 \%$ ). Adverse cardiovascular outcomes were recorded at 30 days.
\end{abstract}

Results: Among 250 patients evaluated by cCTA, 143 (57\%) had no CAD, 64 (26\%) demonstrated minimal plaque ( $<30 \%$ stenosis), $26(10 \%)$ demonstrated mild plaque $(<50 \%$ stenosis), 9 (4\%) demonstrated moderate single vessel disease (50-70\% stenosis), 2 (1\%) demonstrated moderate multivessel disease and $6(2 \%)$ demonstrated severe disease $(>70 \%$ 
stenosis). Six patients developed adverse cardiovascular outcomes. Among traditional cardiac risk factors, only age (older) and sex (male) were significant independent predictors of CAD. Correlation with CAD was poor for TIMI $(\mathrm{r}=0.12)$ and GRACE $(\mathrm{r}=0.09-0.23)$ scores. cCTA identified severe CAD in all subjects with adverse outcomes.

Conclusion: Among ED patients who present with chest pain judged to be at low to intermediate risk of ACS, traditional risk factors are not useful to stratify risk for CAD and adverse outcomes. cCTA is an excellent predictor of CAD and outcome. 


\section{Introduction}

The National Ambulatory Medical Care Survey demonstrates an increasing number of patients presenting annually to the emergency department (ED) with acute chest pain. ${ }^{1}$ Chest pain was the principal reason for over 7 million ED visits in $2009 .^{2}$ As admission of chest pain patients to a coronary care unit is not cost effective, ${ }^{3}$ observation units have been created to improve care and to reduce hospital admissions. ${ }^{4}$ The current practice of observation and diagnostic testing of ED chest pain patients results in billions of dollars in annual medical costs.

Initial assessment of the ED patient with chest pain begins with the medical history, evaluation of cardiac risk factors and assessment of symptoms. The electrocardiogram (ECG), physical examination and cardiac biomarkers may identify higher risk patients. Risk scoring systems - including the Thrombolysis in Myocardial Infarction (TIMI) ${ }^{5}$ and Global Registry of Acute Coronary Events (GRACE) ${ }^{6}$ scores - have been validated to confer additional important prognostic value. ${ }^{7}$ Triage is often based upon myocardial perfusion imaging ${ }^{8}$ or other forms of stress testing. ${ }^{9}$ A scientific statement of the American Heart Association (AHA) supports expedited management of low-risk chest pain patients by combining clinical and laboratory assessments with a confirmatory stress test as "safe, accurate and cost-effective". 10

Conventional standard of care for ED chest pain patients results in a documented miss rate of 2-5\% for acute coronary syndrome (ACS). ${ }^{11,12}$ Missed ACS and subsequent heart attacks account for $20-39 \%$ of all ED malpractice judgments. ${ }^{13}$ Several randomized trials have suggested that coronary CT angiography (cCTA) may be more effective than stress testing for evaluation of low-risk chest pain in the ED. ${ }^{14,15}$ Three randomized multicenter trials have recently confirmed cCTA as a safe and cost-effective diagnostic test to discharge low-risk chest pain patients. ${ }^{16,17,18}$ 
These randomized multicenter studies have opened the possibility of widespread cCTA testing for low risk ED patients presenting with chest pain, though the utility of such testing depends upon the pre-test probability of disease. ${ }^{19}$ Many practicing physicians remain convinced that accurate evaluation of traditional risk factors and clinical presentation can be used for triage of the low risk ED patient and to limit the number of ED patients who should be referred for cCTA. The purpose of the current study was to evaluate individual conventional cardiac risk factors, as well two widely accepted risk scores - TIMI and GRACE - for prediction of coronary artery disease (CAD) and adverse outcomes in an ED population judged to be at low to intermediate risk for ACS. 


\section{Materials and Methods}

This HIPPA compliant clinical protocol was approved by the university institutional review board. Written informed consent was obtained from each participant prior to enrollment in the study. The study was conducted over a 36 month period from August 2009 through July 2012 on consecutive patients who presented to the ED with chest pain or similar symptoms that might represent an anginal equivalent, and who were admitted to the observation unit and evaluated with coronary CTA (cCTA).

\section{Study Population}

The study population included patients presenting to the ED with a chief complaint of chest pain, shortness of breath, syncope or near syncope, or pain radiating to the neck, shoulder, back, or arm and not appearing to be musculoskeletal in nature. Each patient underwent electrocardiography and initial myoglobin and troponin I levels. By agreement with the ED physicians, cCTA was only obtained on patients who they deemed to be at low to intermediate risk of ACS after initial clinical and ECG assessment, and who would otherwise be evaluated with a stress echocardiogram or nuclear perfusion stress test. Patients who were considered high risk for ACS based upon clinical presentation, ECG changes (with clear ST segment elevation or depression) or biomarkers (elevated troponins) were admitted to the hospital or sent directly for cardiac catheterization. Patients with a history of a prior revascularization procedure (bypass surgery or angioplasty \pm stent) were excluded. Patients whose pain was deemed non-cardiac or whose estimated GFR was below $60 \mathrm{ml} / \mathrm{min}$ were excluded. The remaining low to intermediate risk patients were included in the study whenever dedicated cardiac time on the CT scanner was available within 12 hours of presentation. 


\section{History \& Laboratory Data Collection}

In order to prospectively collect information on study participant risk factors, a coinvestigator who was not involved in performance of the cCTA and who was not involved in the care of the patient interviewed each patient with a standard structured questionnaire. This questionnaire was developed in consultation with our cardiologists and ED chest pain division to provide a complete, succinct description of the presenting complaint and cardiac risk factors that could be used for statistical analysis. Chest pain was described by location, character (sharp, aching, burning, tearing, crushing or pressure), radiation (to left arm, jaw/neck, back, right arm, or other), severity (1-10 scale), number of episodes and duration of pain. Patients were asked if pain was exacerbated by exertion or emotional stress. Associated symptoms were recorded. Risk factor evaluation included history of hypertension, hyperlipidemia, diabetes, smoking, prior myocardial infarct, history of known coronary disease, and family history of coronary disease. Medications were recorded and patients were specifically asked about aspirin usage in the previous 7 days. Patient laboratory studies obtained in the ED were reviewed for hyperlipidemia and elevation of troponins. The 12-lead ECG obtained during ED presentation was reinterpreted for study purposes by a cardiology co-investigator. The ED physical examination record was reviewed for body mass index, blood pressure and Killip classification.

\section{Cardiovascular Risk Scores}

The TIMI and GRACE risk scores are commonly used for risk assessment among symptomatic patients with ACS or suspected ACS. For the purpose of this study, an excel spreadsheet was constructed to compute both the TIMI and GRACE scores of our study patients based upon their history and risk factors. 
The TIMI score consists of seven clinical variables with one point given for each condition met. The seven parameters include: age 65 years or older, the presence of at least 3 risk factors for coronary artery disease, a history of prior coronary stenosis greater than or equal to $50 \%$, use of aspirin in the previous 7 days, severe angina defined as experiencing at least 2 episodes of angina within the past 24 hours, ST-segment deviation of at least $0.5 \mathrm{~mm}$ on ECG, and the presence of elevated serum cardiac markers. A patient's TIMI score can easily be calculated during routine clinical evaluation and often serves as the basis for making future medical decisions and obtaining additional medical studies. Details of the TIMI scoring system and a TIMI risk score calculator are available on-line at www.timi.org.

The GRACE score is an alternative risk score to predict outcomes among patients presenting with ACS. The GRACE model utilizes various parameters including a patient's age, Killip class, systolic blood pressure, heart rate, creatinine level, the presence of cardiac arrest on admission, ECG changes (definition is different than the TIMI criteria; ST-segment deviation must be at least $1 \mathrm{~mm}$ ), and elevated serum cardiac markers to generate four probabilities: the probability of death in the hospital and at 6 months from the time of hospital admission, as well as the probability of death or MI in the hospital and at 6 months from the time of hospital admission. In contrast to the TIMI scores, the GRACE score is more complicated to compute and requires use of a calculator. Details of the GRACE scoring system and a GRACE risk score calculator are available on-line at www.outcomes-umassmed.org/grace.

TIMI and GRACE scores were computed based upon the collected data. For the purpose of TIMI and GRACE scores, the ECG recorded in the ED at the time of presentation was reinterpreted by a co-investigator (who is a third year cardiology fellow) using TIMI and GRACE criteria. As many of our patients complained of atypical chest pain or continuous chest 
pain for an extended duration, we contacted the TIMI study group for clarification regarding the definition of severe anginal symptoms. The presence of continuous chest pain over a long interval was tabulated as multiple episodes for the purpose of the TIMI score (personal communication with Dr. Elliott Antman). Four sets of GRACE scores were calculated, including in-hospital and 6 month scores for death and for death/infarction. These research questionnaires, as well as the TIMI and GRACE scores, were not viewed by the ED physicians caring for the patients or by the physician who interpreted the cCTA.

\section{CT Scan Protocol}

Imaging was performed with a 256-MDCT scanner (Brilliance iCT, Philips Medical Systems). Patients with initial heart rates greater than 60 beats/minute were treated with intravenous metoprolol (5-20 mg) to a target heart rate of 50-60 beats/minute. Sublingual nitroglycerin spray $(800 \mu \mathrm{g})$ was administered 2-3 minutes before scanning. Metoprolol was not given to patients with a history of active asthma within the past 30 days or to those with a history

of recent cocaine use. The dose of metoprolol was limited to $10 \mathrm{mg}$ for any patients with a history of asthma. Blood pressure was monitored continuously during premedication, and no further premedication was administered when systolic pressure fell below $100 \mathrm{mmHg}$.

A biphasic injection protocol was used with $60 \mathrm{ml}$ of ioversol (Optiray 350, Mallinckrodt Imaging) followed by $30 \mathrm{ml}$ of ioversol mixed with $30 \mathrm{ml}$ of $0.9 \%$ saline solution, injected at a 5$6 \mathrm{ml} / \mathrm{s}$. Prospective ECG-triggering with axial imaging was used in patients with a stable cardiac rhythm and heart rate $\leq 60$ beats per minute. Retrospective gating with helical imaging and tube current modulation was used in the remaining patients. Tube voltage was set to $120 \mathrm{kVp}$. For prospective axial scans, tube current was set at 60-100mAs based upon patient size. Estimated effective biological dose for patients scanned with prospective ECG-triggering was $4 \mathrm{mSv}$, 
assuming a $12 \mathrm{~cm}$ scan length and a normalization factor of $0.017 \mathrm{mSv} \times \mathrm{mGy}^{-1} \times \mathrm{cm}^{-1}$ for the adult chest. ${ }^{20}$ For helical scans tube current was generally set at $600 \mathrm{mAs} /$ slice, but was increased to 800 or $1000 \mathrm{mAs} /$ slice for obese patients. Estimated mean biological dose for patients scanned at $600 \mathrm{mAs} / \mathrm{slice}$ was $6 \mathrm{mSv}$, assuming a $12 \mathrm{~cm}$ scan length. Imaging was initiated by a dynamic bolus monitoring program which was triggered by contrast enhancement in the left atrium. The CTA was performed during a single breath hold.

\section{CT Image Analysis}

Reconstructed images of the coronary arteries were evaluated by two experienced cardiac radiologists with experience interpreting over 3000 cCTA cases. Disagreements between the two radiologists were resolved by consensus discussion. Slab maximum intensity projection (MIP) images were obtained with $5 \mathrm{~mm}$ slice thickness and rotated in various planes to best visualize any area of stenosis in all coronary segments. For any patient with evidence of stenosis, a tracked reconstruction of the coronary arteries was performed to allow evaluation with a curved multiplanar image and a straightened lumen view (comprehensive cardiac package software; Philips Brilliance workstation. See Figure 1). Each coronary artery segment was rated as normal, minimal disease ( $<30 \%$ stenosis), mild disease ( $<50 \%$ stenosis $)$, moderate disease $(50-70 \%$ stenosis), or severe disease (>70\% stenosis). Based upon these ratings, each cCTA study was classified on a 6 level plaque burden scale $(0=$ no plaque, $1=$ minimal disease with $<30 \%$ stenosis, $2=$ mild disease with $<50 \%$ stenosis, $3=$ moderate single vessel disease with $50-70 \%$ stenosis, $4=$ moderate multivessel disease, and $5=$ severe disease with $>70 \%$ stenosis).

\section{Outcomes \& Clinical Follow-up}


For the purpose of this study, a positive cardiac outcome was defined as confirmed ACS or myocardial infarction, coronary revascularization or cardiac related death within 30 days. A co-investigator attempted to obtain a telephone follow-up on each patient at 30 days following the cCTA. If there was no answer or the patient was unavailable, additional follow-up calls were made in an attempt to reach the patient for up to one year after the cCTA. For those patients who could not be reached by telephone, hospital medical records were searched for follow-up information including subsequent admissions to the hospital, cardiac events, or revascularization. Although the primary endpoint was to identify adverse outcomes at 30 days, in a minority of patients this follow-up data was obtained from telephone conversations or medical records that were recorded up to 1 year after the cCTA.

\section{Statistical Analysis}

Based upon prior experience with cCTA at our institution, we expected to define significant $\mathrm{CAD}$ with $\geq 50 \%$ stenosis in approximately $10 \%$ of our low to intermediate risk ED patients, with adverse outcomes in no more than $2 \%$. As the number of expected adverse outcomes was very small, the study was powered to identify risk factors that might improve the prediction of CAD and allow direct triage of these patients without cCTA. Based upon costeffectiveness considerations, further non-invasive testing for CAD is unlikely to be cost effective when the probability of significant CAD drops below $1 \%$ or rises close to $50 \% .{ }^{19}$ Power calculations were performed assuming a two sample comparison of proportions using a twosided test with an alpha of 0.05 . For a risk factor present in $50 \%$ of the study population, a sample size of 250 is calculated to provide $80 \%$ power to detect a reduction in CAD rate from 
$10 \%$ to $1 \%$, and $85 \%$ power to detect an increase in CAD rate above $25 \%$. For a risk factor that is present in only $20 \%$ of the study population, a sample size of 250 is calculated to provide a power of $70 \%$ to detect an increase in CAD rate above $25 \%$, and $88 \%$ power to detect an increase in CAD rate above $30 \%$.

Statistical analysis was performed by using Stata software (version 12.0, StatCorp). Two sets of dependent variables were used in the analysis, the presence of coronary disease on cCTA and the clinical follow-up outcome data. For the purpose of evaluating the predictive value of risk factors on the presence of coronary disease, significant coronary disease was defined as $\geq$ $50 \%$ stenosis in at least one vessel (a score $\geq 3$ in our grading system). In order to determine whether cCTA was predictive of adverse cardiac outcomes, we cross-tabulated the outcome data with the presence of disease on cCTA.

Univariate analysis: Pearson's correlation coefficients were computed to evaluate correlation of TIMI and GRACE scores with the presence of CAD as demonstrated by cCTA. A chi square test was performed to test the association of binary risk factors (sex, hypertension, hyperlipidemia, diabetes, smoking, etc) with the presence of coronary disease or an adverse cardiac outcome. Logistic regression was used to calculate the odds ratio for the association of binary and continuous risk factors (age, body mass index, severity of pain, TIMI score, GRACE score) with the presence of significant CAD. When multiple categories of a single risk factor were present, as is the case for character of chest pain and radiation of chest pain, an odds ratio was computed for each individual category (ie chest pressure, tightness, etc).

Multivariate analysis: For those risk factors which were found to be significant in the univariate analysis, multivariate logistic regression was performed to determine which of these 
risk factors provided independent significant predictive power for CAD. TIMI and GRACE scores were included in a further multivariate analysis to determine whether they provided additional predictive information. A value of $p<0.05$ was considered statistically significant for all statistical analyses. 


\section{Results}

cCTA studies were obtained on 250 patients evaluated during the study. Demographics of the study population, tabulation of risk factors and odds ratios for prediction of CAD are reported in Table 1. Over half the patients sent for cCTA had no visible coronary plaque ( $\mathrm{n}=143 ; 57 \%$ of subjects). Minimal plaque was present in 64 patients (26\%), mild plaque in 26 patients (10\%), moderate single vessel disease was present in 9 patients (4\%), moderate multivessel disease was present in 2 patients (1\%) and severe plaque in 6 patients (2\%). For the purposes of our analysis, 17 patients were classified as having significant $\mathrm{CAD}$ (stenosis $\geq 50 \%$ ).

Among traditional risk factors, only age (older), sex (male) and hypercholesterolemia were significant predictors of CAD in the univariate analysis (Table 1). The mean age of patients with significant CAD (58.7 years) was significantly greater than the mean age of patients without significant CAD (50.4 years). Other factors including diabetes, hypertension, hypertension on medication, family history, smoking history, history of prior infarction, character of chest pain, radiation of chest pain (to the arm, neck/jaw or back), and severity of chest pain were not significant predictors of CAD. On multivariate analysis, hypercholesterolemia was correlated with age, but was not a significant independent predictor of CAD. Age and sex continued to be significant predictors on multivariate analysis. Of note, 5 of the 7 patients who claimed to have a history of prior myocardial infarction demonstrated no evidence of $\mathrm{CAD}$, and the other 2 had CAD with less than 50\% stenosis on cCTA. Although reinterpretation of the presenting ECG studies by our cardiology co-investigator demonstrated an abnormal result in 17 patients based upon TIMI criteria and 7 patients based upon GRACE criteria (Table 1), only 1 patient with an abnormal ECG result demonstrated significant CAD.

Table 2 tabulates the association of TIMI scores, which varied from 0-5, with the presence of significant CAD on cCTA. Of note, $15 / 16$ patients with coronary stenosis $\geq 50 \%$ had a TIMI score $\leq 2$, while 30/31 patients with a TIMI score $\geq 3$ had no significant coronary stenosis. Table 3 presents the 
Pearson correlation coefficients between risk scores (TIMI and GRACE) and the cCTA grade of CAD, demonstrating only a weak correlation between risk score and plaque burden. Although TIMI and GRACE scores were significantly correlated with each other, the r-values for the correlation of TIMI with the various GRACE scores were in the range of $0.24-0.31(\mathrm{p}<0.001)$.

The data in tables 1, 2 and 3 demonstrate that many patients in our study with significant coronary disease on cCTA are missed by traditional risk factor and/or risk score assessment. Furthermore, a majority of patients with the more elevated TIMI and GRACE scores in this study were, in fact, free of coronary disease. Logistic regression of TIMI scores for prediction of significant CAD with stenosis $\geq$ $50 \%$ failed to demonstrate a significant association. Logistic regression of GRACE scores for prediction of $\mathrm{CAD}$ with stenosis $\geq 50 \%$ did demonstrate a significant association, but the odds ratio was in the range of 1.01-1.03, suggesting weak predictive ability. Furthermore, when any of the GRACE scores was combined with patient age and sex in a multivariate logistic analysis, the GRACE score did not provide additional predictive power for significant $\mathrm{CAD}$ (odds ratios $=0.98-0.99 ; \mathrm{p} \geq 0.4$ ).

Follow-up clinical data were obtained in 221/250 patients, demonstrating 6 adverse cardiovascular events within 30 days. The 6 events included 2 patients with confirmed ACS, 2 patients with myocardial infarction, and 2 patients who required revascularization ( 1 with coronary bypass surgery and 1 with a stent). All 6 adverse cardiovascular events occurred in patients with severe CAD identified by cCTA $($ cCTA grade $=5)$. Although $5 / 6$ patients with adverse events were either hypertensive or diabetic and 2/6 did complain of exacerbation of chest pain with exertion, all 6 adverse cardiovascular events occurred in patients with a TIMI score $\leq 2$ (Table 4). Among the 31 patients with TIMI scores $\geq 3$, none experienced an adverse cardiovascular event on 30 day follow-up. Although all patients who experienced adverse cardiovascular events did have at least one cardiac risk factor, these risk factors were commonly found among patients who did not suffer adverse events, and were not sufficiently specific to triage patients for discharge versus further observation/evaluation. 


\section{Discussion}

Our study confirms the ability of cCTA to predict which patients are at risk for adverse cardiovascular events, as all patients who had an adverse event by 30 days demonstrated the presence of severe CAD on cCTA. Our data also support the conclusion that it is safe to discharge a patient with a normal cCTA or a cCTA showing less than $50 \%$ stenosis, as no adverse outcomes were documented in the 233 patients with less than $50 \%$ coronary stenosis. Furthermore, among conventional criteria used to risk stratify coronary disease, only increasing age and male sex were independent predictors of significant CAD. This finding is concordant with the landmark publication on the probability of CAD by Diamond and Forrester which defined the pre-test likelihood of disease based upon age, sex and presentation of symptoms. ${ }^{21}$ Finally, our data suggest that other commonly employed risk factors are poor predictors for the presence of $\mathrm{CAD}$ in $\mathrm{ED}$ patients, and should not be used to triage low to intermediate risk patients away from cCTA. These findings are concordant with those of the ROMICAT trial subanalyses that demonstrate only "modest" discriminatory capacity of the Goldman, Sanchis and TIMI risk scores for the diagnosis of ACS, as compared with "good" discriminatory capacity for plaque burden on cCTA. ${ }^{22,23}$

Why were the remaining traditional risk factors for CAD and risk scores for adverse outcomes not useful in this low to intermediate risk chest pain population? Numerous studies have validated the application of TIMI and GRACE risk scores to predict patient outcome in the setting of ACS. ${ }^{24}$ For patients presenting to the ED with potential ACS, TIMI and GRACE are the most commonly used scores for risk stratification, with receiver operating characteristic curve (ROC) areas of 0.757 and 0.728 respectively for the prediction of 30 -day event rates. ${ }^{25} \mathrm{~A}$ comparison of TIMI, GRACE and PURSUIT scores concluded that all three risk scores 
demonstrated "good predictive accuracy for death or myocardial infarction at 1 year, and enabled the identification of high-risk subsets of patients who will benefit most from myocardial revascularization performed during initial hospital stay."26

So why did these risk scores fare poorly in our study of patients who presented to the ED with potential ACS? Firstly, we should note that the ROC areas of 0.757 and 0.728 cited above do not suggest excellent predictive value. Furthermore, the comparison of TIMI, GRACE and PURSUIT cited above with "good predictive accuracy" reports ROC areas of 0.551-0.585 for TIMI and 0.672-0.715 for GRACE.

An additional explanation for the poor performance of TIMI and GRACE scores in our study is likely related to the patient population. For patients with confirmed ACS, TIMI and GRACE scores have been validated to predict outcome and distinguish patients who may benefit most from therapy, but no risk score has been validated for identification of ACS in the ED setting. ${ }^{27}$ The low to intermediate risk ED chest pain population is very different from the clinical trial and registry settings of ACS patients in which the TIMI and GRACE scores were developed. Furthermore, although three vessel and left main disease are more common in ACS patients with TIMI scores of 3-4 as compared to patients with TIMI scores of 0-2, significantly more single vessel disease may be present in ACS patients with TIMI scores of 0-2 as compared with those of scores 5-7. ${ }^{28}$ It is likely that the ACS patients who are most commonly seen in the ED are those with single vessel disease and lower risk scores rather than those with extensive CAD and higher risk scores. Our data underscore the importance of risk model validation in an appropriate target population rather than a clinical trial population to establish its generalizability before integration into clinical practice. ${ }^{29}$ 
So how can we best expedite management of the low to intermediate risk patient who presents to the ED with chest pain? Patients who present with chest pain that can be attributed to a non-cardiac cause should require no further cardiac testing. Patients who appear to be at high risk for ACS based upon typical anginal symptoms, ECG findings and biomarkers should be referred for cardiac catheterization. However, the remaining large group of low to intermediate risk patients with atypical chest pain cannot be easily stratified by risk factors and scores. As noted in the introduction, randomized trials have demonstrated excellent negative predictive value for cCTA in evaluation of patients presenting to the ED with chest pain, and have suggested that cCTA may be more cost-effective than traditional stress testing for rapid discharge of these ED chest pain patients. ${ }^{14-18}$ Another significant advantage of cCTA over stress testing is the ability to assess simultaneously for non-cardiac etiologies of chest pain in the mediastinum, lungs and chest wall. In appropriate clinical situations where both cardiac and noncardiac vascular causes of chest pain are suspected, a "triple rule-out" CTA study can evaluate the aorta, coronary arteries and pulmonary arteries and expedite discharge of up to $75 \%$ of patients presenting to the ED with atypical chest pain. ${ }^{30,31}$

A recent editorial in the New England Journal of Medicine critiqued the use of cCTA for low risk ED patients citing the "Choosing Wisely" campaign for diagnostic testing, and declared: "The underlying assumption ... is that some diagnostic test must be performed before discharging these low-to-intermediate-risk patients from the emergency department. This assumption is unproven and probably unwarranted" 32 This editorial appears to oppose the recommendations of the 2010 AHA scientific statement that supports expedited management of low-risk ED chest pain patients by combining clinical assessment with a confirmatory diagnostic test to exclude ischemia as "safe, accurate and cost-effective". ${ }^{10}$ Our study suggests that it would 
be unwise to discharge low to intermediate risk ED patients on the basis of risk factors and scores without confirmatory testing, as cCTA did identify a small percentage of patients with significant CAD $(17 / 250=6.8 \%)$ and 30 day adverse events $(6 / 221=2.7 \%)$ who might not otherwise be detected based upon clinical presentation to the ED. It is also unlikely that our ED physicians would be willing to discharge low to intermediate risk patients when missed CAD/ACS and subsequent heart attacks account for 20-39\% of all ED malpractice judgments. ${ }^{13}$ A recent analysis by the Physician Insurers Association of America states "Between 1985 and 2012, the third most common patient condition for which claims were filed against emergency physicians were Acute Myocardial Infarctions (AMIs) . . Claims involving AMIs resulted with an indemnity payment $52 \%$ of the time with an average payment of $\$ 261,856 "{ }^{33}$

Several limitations of our study design should be noted. The accuracy of risk factor assessment depends upon accurate recording of risk factors, and patients are often uncertain of their own history or unable to adequately explain their symptoms. This uncertainty is illustrated by the 7 patients who claimed to have had a prior myocardial infarction, but who had no significant coronary disease on cCTA. Furthermore, clinical history and risk factor assessment is often quite variable in the ED setting. For the current study, we attempted to address this issue by prospectively interviewing all patients with a standardized history and risk factor questionnaire that included all standard risk factors as well as all data needed to compute TIMI and GRACE risk scores. This questionnaire was based upon an existing chest pain assessment form which was modified after discussions with our cardiologists and ED observation unit physicians, and expanded to incorporate additional information required for TIMI and GRACE scores. When there was uncertainty regarding a risk factor, we checked back with the medical records and ED chart. With respect to the follow-up data, we initially wanted to collect all follow-up data at 30 
days. However, because we were unable to contact many patients within this time interval, the telephone follow-up period was expanded to increase the follow-up rate, and additional followup was obtained from medical records. Nonetheless, 30 day follow-up data could not be obtained for 29 patients, representing $11.6 \%$ of the patients involved in the study. Furthermore, although 30 day follow-up data should be sufficient to demonstrate the safety of ED discharge, this short term follow-up is not sufficient to demonstrate the long-term prognostic utility of cCTA. Finally, as the focus of this study was upon CAD and cardiovascular outcomes, the 30 day follow-up was focused upon cardiac events but we did not tabulate the non-cardiac pathology detected by cCTA, nor did we obtain follow-up for non-cardiac adverse events.

The low rate of adverse outcomes among ED patients is a limitation for statistical analysis of the risk factors, and might interfere with detection of statistically significant risk factors. Considering those variables with odds ratios $>1.5$ in table 1 , it is possible that a larger study population would have found statistical significance in the risk factors of hypertension, diabetes, a description of tearing chest pain, and exacerbation of chest pain with exertion. Nonetheless, our outcome results demonstrate that these risk factors did not have good discriminating ability for the limited number of adverse cardiovascular outcomes in our study population. In order to improve our statistical power to demonstrate significant risk factors, we chose to analyze the predictive value of traditional cardiac risk factors and risk scores for both CAD and clinical outcomes. Some degree of CAD was present in $43 \%$ of the study population, and significant $\mathrm{CAD}$ with greater than $50 \%$ stenosis was present in $7 \%$ of the study population. Since the development of ACS requires the presence of underlying coronary disease, CAD is reasonable as a proxy marker for potential future development of an adverse cardiovascular event. 
Despite these limitations, our data demonstrate that among patients who present to the ED with chest pain and are assessed to be at low to intermediate risk of ACS, traditional risk factors, TIMI and GRACE scores do not predict plaque burden on cCTA or adverse cardiovascular outcomes. cCTA is an effective strategy to triage these patients, but risk factors cannot be used to decide which patients should have cCTA in the low to intermediate risk group seen in our ED. Specifically, cCTA identified patients with significant CAD who would be missed by evaluation of risk factors, TIMI and GRACE scores. cCTA expedited the discharge of over $75 \%$ of patients - even those with higher risk scores - based upon normal or minimal disease in the coronary arteries. In conclusion, for a patient who presents to the ED with chest pain and is assessed to be at low to intermediate risk of ACS, cCTA is an appropriate diagnostic study and is superior to clinical assessment by risk factors and risk scores for patient triage.

Figure caption:

Figure 1. Coronary artery grading system for stenosis, demonstrating curved multiplanar and straightened lumen views of the left anterior descending artery (LAD) in 6 patients.
A. Normal LAD
B. Minimal disease (arrow) with $<30 \%$ stenosis.
C. Mild disease (arrow) with $<50 \%$ stenosis.
D. Moderate disease (arrow) with 50-70\% stenosis.
E. Severe disease (arrow) with $>70 \%$ stenosis. Follow-up: treated with stent, symptoms resolved.
F. Severe disease (arrow) with $>70 \%$ stenosis, and more proximal disease with moderate $50-70 \%$ stenosis (arrowhead). Follow-up: Sent to cath lab for recurrent symptoms, and diagnosed with ACS. 


\section{Figures:}

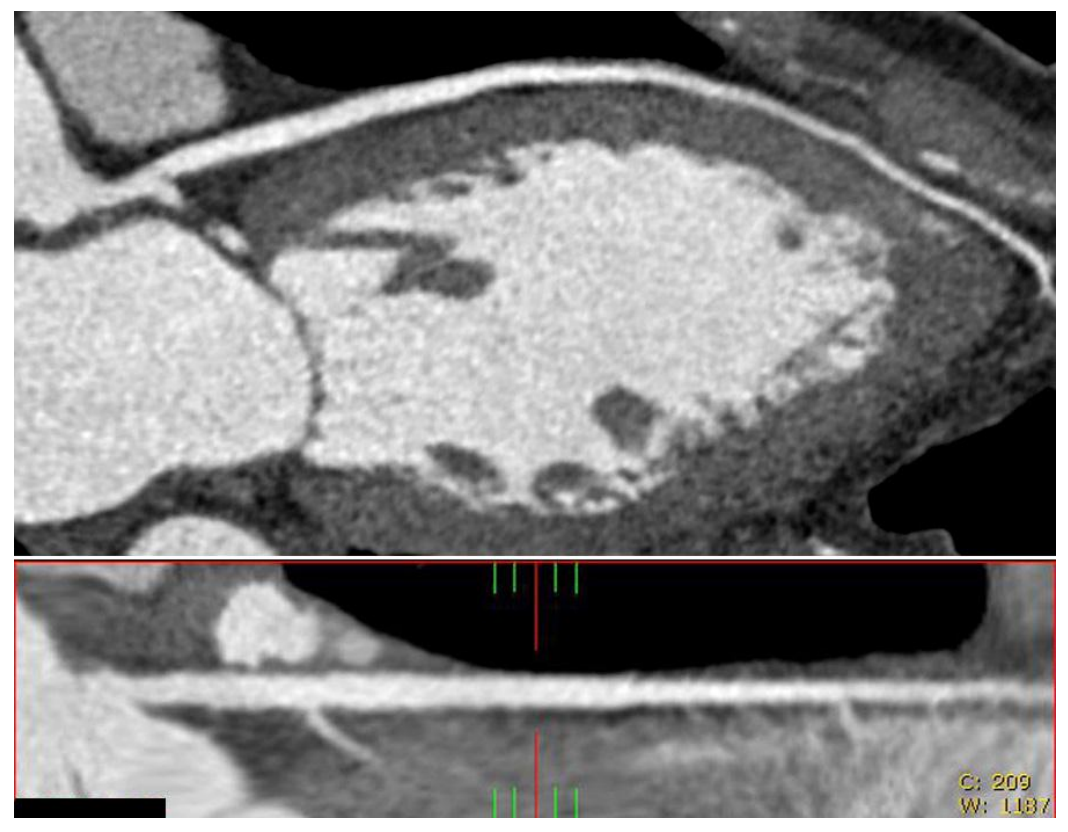

Figure 1. Coronary artery grading system for stenosis, demonstrating curved multiplanar and straightened lumen views of the left anterior descending artery (LAD) in 6 patients.

\section{A. Normal LAD}

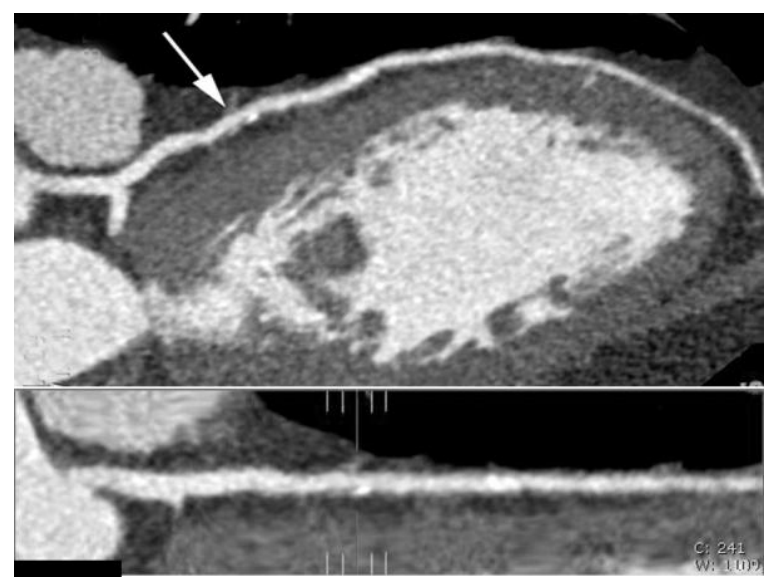

Figure 1. Coronary artery grading system for stenosis, demonstrating curved multiplanar and straightened lumen views of the left anterior descending artery (LAD) in 6 patients.

B. Minimal disease (arrow) with $<30 \%$ stenosis. 


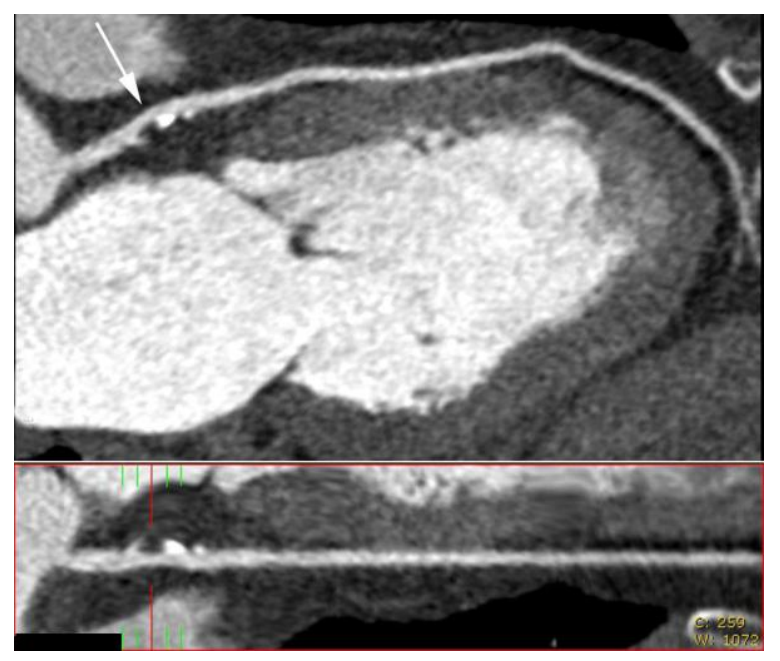

Figure 1. Coronary artery grading system for stenosis, demonstrating curved multiplanar and straightened lumen views of the left anterior descending artery (LAD) in 6 patients.

C. Mild disease (arrow) with $<50 \%$ stenosis.

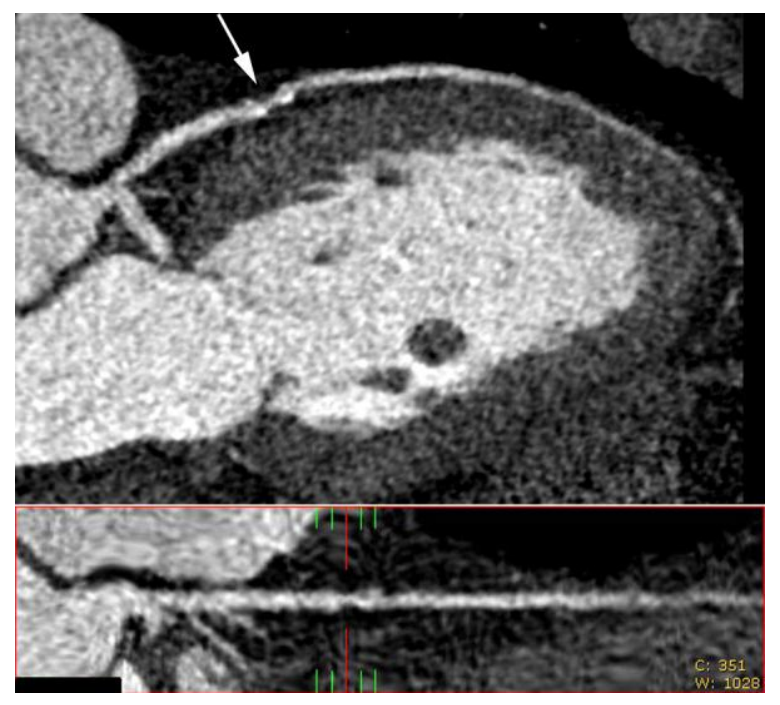

Figure 1. Coronary artery grading system for stenosis, demonstrating curved multiplanar and straightened lumen views of the left anterior descending artery (LAD) in 6 patients.

D. Moderate disease (arrow) with 50-70\% stenosis. 


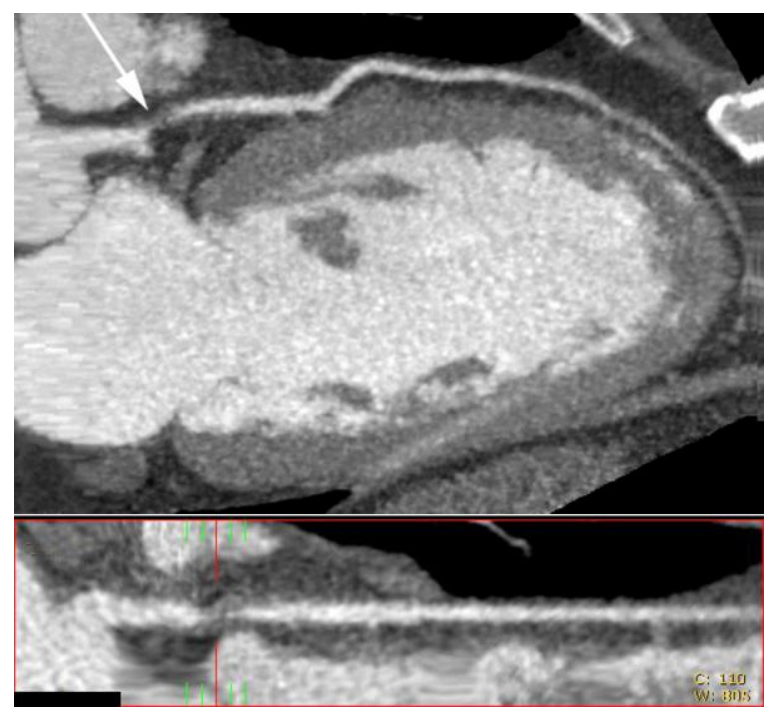

Figure 1. Coronary artery grading system for stenosis, demonstrating curved multiplanar and straightened lumen views of the left anterior descending artery (LAD) in 6 patients.

E. Severe disease (arrow) with $>70 \%$ stenosis. Follow-up: treated with stent, symptoms resolved.

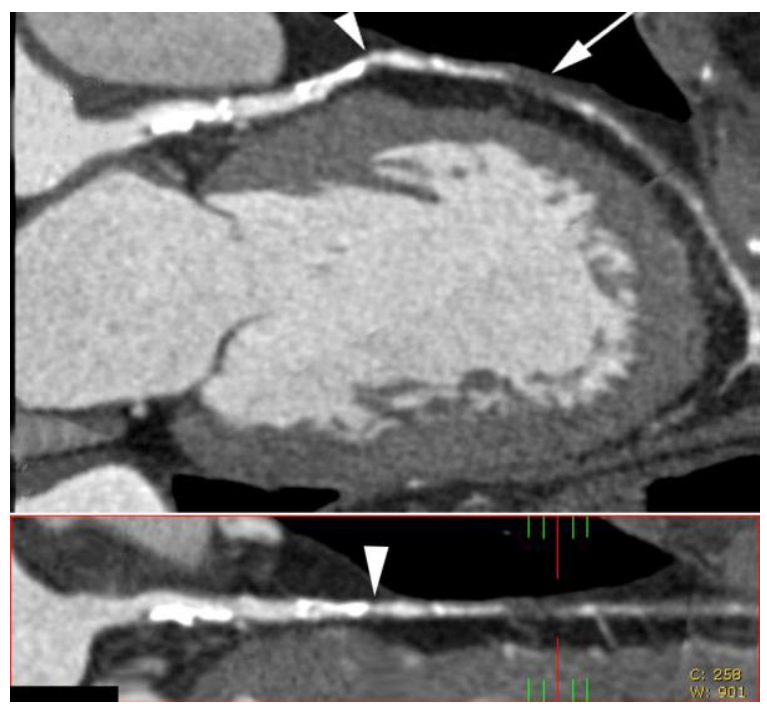

Figure 1. Coronary artery grading system for stenosis, demonstrating curved multiplanar and straightened lumen views of the left anterior descending artery (LAD) in 6 patients.

F. Severe disease (arrow) with $>70 \%$ stenosis, and more proximal disease with moderate $50-70 \%$ stenosis (arrowhead). Follow-up: Sent to cath lab for recurrent symptoms, and diagnosed with ACS. 


\section{References}

${ }^{1}$ Niska R, Bhuiya F, Xu J. National Hospital Ambulatory Medical Care Survey: 2007 emergency department summary. National Health Statistics Report \#26, 8/6/2010.

${ }^{2}$ National Hospital Ambulatory Medical Care Survey: 2009 Emergency Department Summary Tables. See http://www.cdc.gov/nchs/data/ahcd/nhamcs_emergency/2009_ed_web_tables.pdf

${ }^{3}$ Tosteson AN, Goldman L, Udvarhelyi IS, Lee TH. Cost-effectiveness of a coronary care unit versus an intermediate care unit for emergency department patients with chest pain. Circulation. 1996; 94: 143-50.

${ }^{4}$ Venkatesh AK, Geisler BP, Gibson Chambers JJ, Baugh CW, Bohan JS, Schuur JD. Use of observation care in US emergency departments, 2001 to 2008. PLoS One. 2011;6(9):e24326. Epub 2011 Sep 14. Erratum in: PLoS One. 2012;7(1). doi: 10.1371/annotation/e7927237-fcce-4738-9564-e9f5d85341cb.

${ }^{5}$ Antman EM, Cohen M, Bernink PJ, McCabe CH, Horacek T, Papuchis G, Mautner B, Corbalan R, Radley D, Braunwald $\mathrm{E}$. The TIMI risk score for unstable angina/non-ST elevation MI: A method for prognostication and therapeutic decision making. JAMA 2000 Aug 16;284(7):835-42.

${ }^{6}$ The GRACE Investigators. Rationale and design of the GRACE (Global Registry of Acute Coronary Events) project: a multinational registry of patients hospitalized with acute coronary syndromes. Am Heart J 2001;141:190-9.

${ }^{7}$ Yan AT, Yan RT, Tan M, Casanova A, Labinaz M, Sridhar K, Fitchett DH, Langer A, Goodman SG. Risk scores for risk stratification in acute coronary syndromes: useful but simpler is not necessarily better. Eur Heart J 2007.

${ }^{8}$ Kontos MC, Schmidt KL, McCue M, Rossiter LF, Jurgensen M, Nicholson CS, Jesse RL, Ornato JP, Tatum JL. A comprehensive strategy for the evaluation and triage of the chest pain patient: a cost comparison study. J Nucl Cardiol. 2003 May-Jun;10(3):284-90.

${ }^{9}$ Chandra A, Rudraiah L, Zalenski RJ. Stress testing for risk stratification of patients with low to moderate probability of acute cardiac ischemia. Emerg Med Clin North Am. 2001 Feb;19(1):87-103.

${ }^{10}$ Amsterdam EA, Kirk JD, Bluemke DA, Diercks D, Farkouh ME, Garvey JL, Kontos MC, McCord J, Miller TD, Morise A, Newby LK, Ruberg FL, Scordo KA, Thompson PD; American Heart Association Exercise, Cardiac Rehabilitation, and Prevention Committee of the Council on Clinical Cardiology, Council on Cardiovascular Nursing, and Interdisciplinary Council on Quality of Care and Outcomes Research. Testing of low-risk patients presenting to the emergency department with chest pain: a scientific statement from the American Heart Association. Circulation. 2010 Oct 26;122(17):1756-76.

${ }^{11}$ Pope JH, Aufderheide TP, Ruthazer R, Woolard RH. Feldman JA, Beshansky JR, Griffith JL, Selker HP. Missed diagnoses of acute cardiac ischemia in the emergency department. N Engl J Med 2000; 342: 1163-70.

${ }^{12}$ Schull M, Vermeulen MJ, Stukel TA. The risk of missed diagnosis of acute myocardial infarction associated with emergency department volume. Ann Emerg Med 2006; 48: 647-55.

${ }^{13}$ Storrow AB, Gibler WB. Chest pain centers: diagnosis of acute coronary syndromes. Ann Emerg Med 2000; 35 : 449-61

${ }^{14}$ Goldstein JA, Gallagher MJ, O'Neill WW, Ross MA, O'Neil BJ, Raff GL. A randomized controlled trial of multislice coronary computed tomography for evaluation of acute chest pain. J Am Coll Cardiol. 2007 Feb 27;49(8):86371.

${ }^{15}$ Miller AH, Pepe PE, Peshock R, Bhore R, Yancy CC, Xuan L, Miller MM, Huet GR, Trimmer C, Davis R, Chason R, Kashner MT. Is coronary computed tomography angiography a resource sparing strategy in the risk 
stratification and evaluation of acute chest pain? Results of a randomized controlled trial. Acad Emerg Med. 2011 May;18(5):458-67.

${ }^{16}$ Goldstein JA, Chinnaiyan KM, Abidov A, Achenbach S, Berman DS, Hayes SW, Hoffmann U, Lesser JR, Mikati IA, O'Neil BJ, Shaw LJ, Shen MY, Valeti US, Raff GL; CT-STAT Investigators. The CT-STAT (Coronary Computed Tomographic Angiography for Systematic Triage of Acute Chest Pain Patients to Treatment) trial. J Am Coll Cardiol. 2011 Sep 27;58(14):1414-22.

${ }^{17}$ Litt HI, Gatsonis C, Snyder B, Singh H, Miller CD, Entrikin DW, Leaming JM, Gavin LJ, Pacella CB, Hollander JE. CT angiography for safe discharge of patients with possible acute coronary syndromes. N Engl J Med. $2012 \mathrm{Apr}$ 12;366(15):1393-403.

${ }^{18}$ Hoffmann U, Truong QA, Schoenfeld DA, Chou ET, Woodard PK, Nagurney JT, Pope JH, Hauser TH, White CS, Weiner SG, Kalanjian S, Mullins ME, Mikati I, Peacock WF, Zakroysky P, Hayden D, Goehler A, Lee H, Gazelle GS, Wiviott SD, Fleg JL, Udelson JE; ROMICAT-II Investigators. Coronary CT angiography versus standard evaluation in acute chest pain. N Engl J Med. 2012 Jul 26;367(4):299-308.

${ }^{19}$ Halpern EJ, Fischman D, Savage M, Koka AR, DeCaro M, Levin DC. Decision analytic model for evaluation of suspected coronary disease with stress testing and coronary CT angiography. Acad Radiol. 2010; 17(5):577-86.

${ }^{20}$ McCollough C, Cody D, Edyvean S, et al. The measurement, reporting, and management of radiation dose in CT. College Park, MD: American Association of Physicists in Medicine, 2008: AAPM report no. 96

${ }^{21}$ Diamond GA, Forrester JS. Analysis of probability as an aid in the clinical diagnosis of coronary-artery disease. N Engl J Med 1979; 300(24):1350-1358.

${ }^{22}$ Manini AF, Dannemann N, Brown DF, Butler J, Bamberg F, Nagurney JT, Nichols JH, Hoffmann U; Rule-Out Myocardial Infarction using Coronary Artery Tomography (ROMICAT) Study Investigators. Limitations of risk score models in patients with acute chest pain. Am J Emerg Med. 2009;27(1):43-8.

${ }^{23}$ Ferencik M, Schlett CL, Bamberg F, Truong QA, Nichols JH, Pena AJ, Shapiro MD, Rogers IS, Seneviratne S, Parry BA, Cury RC, Brady TJ, Brown DF, Nagurney JT, Hoffmann U. Comparison of traditional cardiovascular risk models and coronary atherosclerotic plaque as detected by computed tomography for prediction of acute coronary syndrome in patients with acute chest pain. Acad Emerg Med. 2012; 19(8):934-42.

${ }^{24}$ D'Ascenzo F, Biondi-Zoccai G, Moretti C, Bollati M, Omedè P, Sciuto F, Presutti DG, Modena MG, Gasparini M, Reed MJ, Sheiban I, Gaita F. TIMI, GRACE and alternative risk scores in Acute Coronary Syndromes: a metaanalysis of 40 derivation studies on 216,552 patients and of 42 validation studies on 31,625 patients. Contemp Clin Trials. 2012 May;33(3):507-14.

${ }^{25}$ Lee B, Chang AM, Matsuura AC, Marcoon S, Hollander JE. Comparison of cardiac risk scores in ED patients with potential acute coronary syndrome. Crit Pathw Cardiol. 2011 Jun;10(2):64-8.

${ }^{26}$ de Araújo Gonçalves P, Ferreira J, Aguiar C, Seabra-Gomes R. TIMI, PURSUIT, and GRACE risk scores: sustained prognostic value and interaction with revascularization in NSTE-ACS. Eur Heart J. 2005 May;26(9):86572.

${ }^{27}$ Backus BE, Six AJ, Kelder JH, Gibler WB, Moll FL, Doevendans PA. Risk scores for patients with chest pain: evaluation in the emergency department. Curr Cardiol Rev. 2011 Feb;7(1):2-8. 
${ }^{28}$ Garcia S, Canoniero M, Peter A, de Marchena E, Ferreira A. Correlation of TIMI risk score with angiographic severity and extent of coronary artery disease in patient with non-ST-elevatgion acute coronary syndromes. Am J Cardiol. 2004 Apr 1;93(7):813-6.

${ }^{29}$ Yan AT, Jong P, Yan RT, Tan M, Fitchett D, Chow CM, Roe MT, Pieper KS, Langer A, Goodman SG. Clinical trial--derived risk model may not generalize to real-world patients with acute coronary syndrome. Am Heart J 2004;148:1020-1027.

${ }^{30}$ Halpern EJ. Triple-rule-out CT angiography for evaluation of acute chest pain and possible acute coronary syndrome. Radiology. 2009 Aug;252(2):332-45.

${ }^{31}$ Takakuwa KM, Halpern EJ, Shofer FS. A time and imaging cost analysis of low-risk ED observation patients: a conservative 64-section computed tomography coronary angiography "triple rule-out" compared to nuclear stress test strategy. Am J Emerg Med. 2011 Feb;29(2):187-95.

${ }^{32}$ Redberg RF. Coronary CT angiography for acute chest pain. N Engl J Med. 2012 Jul 26;367(4):375-6.

${ }^{33} 2012$ Risk Management Review - Emergency Medicine. Physician Insurers Association of America (PIAA). 